\title{
Red Cell Distribution Width Predicts the Left Ventricular Reverse Remodeling After ST Segment Elevation Myocardial Infarction Undergone Primary Percutaneous Coronary Intervention
}

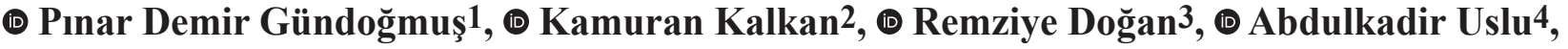 \\ (1) Enbiya Aksakal3
}

129 May1s State Hospital, Clinic of Cardiology, Ankara, Turkey

2Erzurum Training and Research Hospital, Clinic of Cardiology, Erzurum, Turkey

${ }^{3}$ Atatürk University Medical School, Heart Center, Department of Cardiology, Erzurum, Turkey

${ }^{4}$ Kartal Koşuyolu Training and Research Hospital, Clinic of Cardiology, İstanbul, Turkey

\begin{abstract}
Objectives: Previous studies have shown that red blood cell distribution width (RDW) is associated with some cardiac diseases. We investigated whether RDW was associated with left ventricular (LV) reverse remodeling after ST segment elevation myocardial infarction.

Materials and Methods: This retrospective study analyzed 300 patients with ST segment elevation myocardial infarction who underwent a follow-up echocardiography. We recorded the clinical characteristics, laboratory values

and echocardiographic signs of the patients on admission and in the third month. The patients were categorized into two groups according to echocardiographic assessment after 3 months: LV reverse remodeling and non-reverse remodeling.

Results: The RDW levels were significantly lower in the patients who had LV reverse remodeling than the patients who had non-reverse remodeling (15.4 \pm 2 vs $17.8 \pm 3.6$, $\mathrm{p}<0.001)$. In the multivariate logistic regression analysis,
\end{abstract}

Address for Correspondence: Pınar Demir Gündoğmuş, 29 Mayıs State Hospital, Clinic of Cardiology, Ankara, Turkey Phone: +90 5397272561 e-mail: 1pinar.demir@gmail.com ORCID: orcid.org/0000-0001-8042-189X Received: 10.01.2020 Accepted: 09.04.2020

Cite this article as: Demir Gündoğmuş P, Kalkan K, Doğan R, Uslu A, Aksakal E. Red Cell Distribution Width Predicts the Left Ventricular Reverse Remodeling After ST Segment Elevation Myocardial Infarction Undergone Primary Percutaneous Coronary Intervention. EJCM 2020;8(2):63-71.

DOI: $10.32596 /$ ejcm.galenos.2020.01.01

${ }^{\circ}$ Copyright 2020 by Heart and Health Foundation of Turkey (TÜSAV) / E Journal of Cardiovascular Medicine published by Galenos Publishing House. 


\begin{abstract}
the independent predictors of reverse remodeling were Diabetes Mellitus $(\mathrm{p}=0.001)$, history of coronary artery bypass grafting surgery $(\mathrm{p}=0.031)$, ST segment resolution $(\mathrm{p}=0.021)$, Thrombolysis in myocardial infarction flow classification $(\mathrm{p}=0.023)$ and RDW level $(\mathrm{p}<0.001)$. According to the receiver operating characteristic curve analysis, it was found that RDW (for the 17.3 level) had an
\end{abstract}

\section{Introduction}

Heart failure (HF), which occurs mostly as postmyocardial infarction, is still one of the leading causes of mortality and morbidity in the world ${ }^{(1)}$. After acute myocardial infarction (AMI), secondary to functional myocardial injury, the myocardium receives adaptive responses to save the same contractile function. As a result of these responses, in the broadest sense, left ventricular (LV) remodeling is a general term used to define an interstitial, molecular and genetic change that occurs by clinical changes in size, shape and function of LV after a cardiac injury ${ }^{(2)}$. LV remodeling after AMI is accepted as an important indicator of the prognosis and development of $\mathrm{HF}^{(3)}$. The physiology of LV remodeling includes the size of the infarction area, oxidative stress, collagen synthesis, cytokine activation, ventricular dilatation, ventricular hypertrophy, scar formation and neurohormonal responses ${ }^{(4)}$. The term reverse remodeling is defined as a process that involves the regression of pathological myocardial hypertrophy, chamber shape distortions and dysfunction ${ }^{(5)}$. In other words, reverse remodeling is defined as myocardial tissue damaged by various factors, which initiates the healing process by stopping the remodeling process, as a result of various therapeutic treatments or elimination of injuring factors. Major guideline-directed HF therapies such as angiotensin converting enzyme inhibitors and beta blockers target the neurohormonal pathways in attempts to prevent remodeling and provide reverse remodeling ${ }^{(6)}$. Revascularization estimate for LV reverse remodeling.

Conclusion: We suggest that the RDW level that is counted during routine complete blood count test might be useful to estimate LV reverse remodeling.

Keywords: Myocardial infarction, reverse remodeling, red blood cell distribution width (RDW), STEMI

therapies [coronary artery bypass grafting surgery $(\mathrm{CABG})$ and primary percutaneous coronary intervention (PPCI)] aim to decrease ischemia time and limit myocardial injury and thus support the reverse remodeling process $^{(7)}$. LV reverse remodeling plays a key role for improving LV function and myocardial recovery.

Red blood cell distribution width (RDW) is obtained with a basic hemogram test in clinical practice, it is a quantitative measure of the size variability of circulating erythrocytes, and values above normal indicate a more heterogeneous population of cells ${ }^{(8)}$. Recent studies have shown that RDW may be an important predictor of cardiovascular disease progression and inflammatory process $^{(9)}$. Recently, more focus has been on the predictive and prognostic value of RDW in patients with coronary heart disease, HF, atrial fibrillation and acute pulmonary embolism $^{(10-12)}$. However, even though RDW has been linked with an increased risk of adverse outcomes in patients with HF, there are a few studies to show its relationship with the severity of LV dysfunction, and surprisingly, little is known about the correlation between RDW and the prognosis of LV dysfunction ${ }^{(13,14)}$. We investigated whether RDW was associated with LV reverse remodeling after ST segment elevation myocardial infarction (STEMI).

\section{Materials and Methods}

\section{Population}

The participants were retrospectively recruited from the cardiology clinic at the Atatürk University Training 
and Research Hospital between September 2014 and September 2015. A total of 300 patients (239 males, mean age: $57.4 \pm 10.5$ years) with the diagnosis of STEMI, who underwent PPCI of the culprit lesion after coronary angiography, were included. STEMI was diagnosed according to the criteria of the European Society of Cardiology in $2018^{(15)}$. The inclusion criteria were as follows: 1) having chest pain having started in the last 12 hours; 2) undergoing PPCI during coronary angiography; 3 ) being older than 18 years. On the other hand, patients with anemia (hemoglobin $<13 \mathrm{gr} / \mathrm{dL}$ for men, $<12 \mathrm{gr} / \mathrm{dL}$ for women), clinical evidence of cancer, active infection, active or chronic inflammatory or autoimmune diseases, pregnancy, known history of HF or previously LVEF < $35 \%$, and those using steroids were excluded from the study.

The study protocol was approved by the Clinical Trials Ethics Committee of Atatürk University (decision no and date: $4 / 17$ and 02.06.2015).

\section{Procedure}

All patients' age, gender, medical history, risk factors [smoking, Diabetes Mellitus (DM), hypertension (HT) etc.], family history, presence of comorbidities (anemia, chronic renal insufficiency, chronic obstructive pulmonary disease), medications, clinical and laboratory data were recorded. Chest pain duration and Killip classes were determined at the time of admission ${ }^{(16)}$.

Complete blood counts and biochemical values were recorded for all patients. Hematological indices (hemoglobin, number of white cells, number of platelets and red cell distribution width) were measured and recorded using a Coulter LH 780 Hematology Analyzer (Beckman Coulter Ireland Inc., Mervue, Galway, Ireland). Percutaneous coronary intervention (stenting and/or balloon angioplasty) was performed on the culprit lesion for coronary angiography after the transfemoral approach using the standard Judkins method. Infarction-related arteries were classified as the left anterior descending artery (LAD), circumflex artery, right coronary artery, saphenous graft and others. Coronary blood flow was detected by Thrombolysis in Myocardial Infarction (TIMI) classification on the angiographic images after PPCI ${ }^{(17)}$.

Echocardiographic evaluations of the patients were made within the first 24-48 hours and 3 months after hospitalization. The patients were imaged by using GE Vivid 7 (Vivid Seven, General Electric-Vingmed, Milwaukee, WI, USA), which was equipped with a transducer employing harmonic imaging. M-mode echocardiographic measurements were on the basis of the standards. Echocardiograms were taken from the left lateral decubitus position and from the standard echocardiographic windows (parasternal long and short axis, apical 2 and 4 cavities). The LVEF values of the patients were assessed using the Teichhold, Biplane Simpson methods or visually. LVEF, LV systolic and diastolic end-point measurements and interventricular septum measurements were recorded. At the end of three months of STEMI, those with an increase in LVEF by $5 \%$ and higher on echocardiography were defined as the group with LV reverse remodeling. The group with an increase under 5\% in LVEF was assessed as the group with LV non-reverse remodeling ${ }^{(18,19)}$.

\section{Statistical Analysis}

In statistical analysis, the numerical variables were presented as mean \pm standard deviation, while the categorical variables were expressed as percentages. The Kolmogorov-Smirnov test was used to determine if the numerical variables were normally distributed. The Student's t-test was used to compare the normally distributed numerical variables between the groups, and the Mann-Whitney U test was used to compare the nonnormally distributed numerical variables between the groups. The chi-square test was employed to compare the categorical variables between the groups. The Spearman's or Pearson's correlation analysis was used in the analysis of the relationship between the numerical variables. Variables with a $\mathrm{p}$ value of $<0.100$ in the univariate analyses between the patients who had LV reverse remodeling and LV non-reverse remodeling on a mean 
follow-up of three months were subjected to multivariate analysis to determine the independent determinants of LV reverse remodeling. Additionally, ROC curve analysis was performed to determine which value of RDW had the best sensitivity and specificity for estimating LV reverse remodeling. In all statistical analyses, $p<0.05$ was accepted as the limit of significance. Statistical analyses were performed using the SPSS 20.0 (SPSS Inc, Chicago, IL, USA) package program.

\section{Results}

A total of 300 patients with STEMI were included in the study. The mean age of the patients was $57.4 \pm 10.5$ years, and $79.7 \%$ were male. The rate of the patients with HT was $43.3 \%$, and the rate of the patients with DM was $29 \%$. The rate of the patients who smoked was $57.7 \%$, and the rate of patients with dyslipidemia was $40.3 \%$. The rate of the patients with previous history of AMI was $11.7 \%$. $13.3 \%$ of the patients were using aspirin, $16.7 \%$ were using oral antidiabetics, and $20.3 \%$ were using statin. The proportion of the patients with three vessel diseases was $12.3 \%$, and the infarction-related artery was detected as $46.3 \%$ of $\mathrm{LAD}$. The mean RDW value of the patients was 16.5 \pm 3.2 . The mean baseline LVEF value was $46.7 \pm 7.5$, and the mean EF value after 3 months was $49.5 \pm 9.5$ (Table 1).

Comparisons between the groups showed a significantly lower incidence of DM $(17.3 \%$ vs $39.1 \%, \mathrm{p}<0.001)$ in the LV reverse remodeling group. The glucose levels were lower in the LV reverse remodeling group in the comparison of the groups ( $143 \pm 69$ vs $161 \pm 90, \mathrm{p}=0.04)$. The mean RDW value was lower in the LV reverse remodeling group than in the non-reverse remodeling group $(15.4 \pm 2.4$ vs $17.8 \pm 3.6, \mathrm{p}<0.001)$ (Figure 1). At the end of three months, the patients' LVEF values were significantly higher in the LV reverse remodeling group (53.9 \pm 7.8 vs $45.6 \pm 9, p<0.001)$ (Table 1$)$.

In the analyses between the patients in the LV reverse remodeling group and non-reverse remodeling group on a mean follow-up of three months, the variables with a $\mathrm{p}$ value $<0.100$ were analyzed with multivariate analysis to determine the independent predictors of LV reverse remodeling. In the multivariate analysis that was performed, the presence of DM [Odds ratio (OR): 3.4, 95\% confidence interval $(\mathrm{CI}): 1.7-6.7, \mathrm{p}=0.001]$, history of CABG (OR: 11.9, 95\% CI: 1.2-13.2, $\mathrm{p}=0.031$ ), ST segment resolution (OR: 0.514, 95\% CI 0.29-0.9, $\mathrm{p}=0.021$ ), the classes of TIMI (OR: 0.39, 95\% CI: $0.18-0.87, \mathrm{p}=0.023$ ) and RDW values (OR: $1.30,95 \%$ CI: $1.20-1.43, \mathrm{p}<0.001)$ were determined as the independent predictors of LV reverse remodeling (Table 2).

Additionally, ROC curve analysis was performed to determine which level of RDW had the best sensitivity and specificity to predict $L V$ reverse remodeling. As a result of the ROC curve analysis, the AUC value was 0.722 (0.654$0.789, \mathrm{p}<0.001)$. The predictive value of RDW for reverse remodeling, with $71 \%$ sensitivity and $79 \%$ specificity, was found to be 17.3 (Figure 2).

\section{Discussion}

One of the major results of our study was that the RDW levels on admission predicted the LV reverse remodeling in patients with STEMI undergoing PPCI. A cut-off value of 17.3 for RDW was obtained to predict LV reverse remodeling after a 3-month follow-up. As far as we know, this is the first study in the literature to show a relationship between RDW and LV reverse remodeling. Moreover, other results of the study on the presence of DM, CABG operation history, ST segment depression rate after PPCI and TIMI flow class may be used in STEMI patients as a predictor of LV reverse remodeling. Moreover, this study found that DM, smoking, ST segment resolution, TIMI risk score and glucose levels were associated with LV remodeling development in the patient population of study. It is possible to say that these data were in accordance with the literature.

The most important consequence of morbidity and mortality affecting quality of life in the long term after STEMI is the development of HF as a result of LV systolic 
Table 1. The clinical, laboratory and echocardiographic characteristics of patient groups without and with left ventricular remodeling

Age, years (mean \pm SD)

Male, \%

Arterial hypertension, \%

Diabetes Mellitus, \%

Smoking, \%

Hyperlipidemia, (\%)

Family history of ischemic heart disease, \%

History of myocardial infarction, (\%)

History of $\mathrm{PCl}$, (\%)

History of CABG operation, \%

ASA using, $\%$

Clopidogrel using, \%

Oral antidiabetic using, $\%$

Beta-blockers using, \%

Statins using, \%

ACE inhibitors using, $\%$

Ca channel blockers using, $\%$

Killip classification 3-4, \%

ST segment resolution (0-30), \%

Three vessel disease, $\%$

Infarct related artery, \%

LDA

CX

RCA

TIMI risk score $3 \%$

Door-balloon time (mean \pm SD)

Systolic blood pressure, $\mathrm{mmHg}$ (mean \pm SD)

Diastolic blood pressure, $\mathrm{mmHg}$ (mean $\pm \mathrm{SD}$ )

Heart rate, pulse/min (mean $\pm S D$ )

WBC $10^{3} / \mathrm{mL}$ (mean \pm SD)

Hemoglobin, g/dL (mean \pm SD)

RDW (mean \pm SD)

Platelets $10^{3} / \mathrm{mL}$ (mean \pm SD)

Creatinine (mean \pm SD)

Glucose, mg/dL (mean \pm SD)

Total cholesterol, $\mathrm{mg} / \mathrm{dL}$ (mean $\pm \mathrm{SD}$ )

LDL-cholesterol, mg/dL (mean \pm SD)

HDL-cholesterol, $\mathrm{mg} / \mathrm{dL}$ (mean $\pm \mathrm{SD}$ )

Triglyceride, $\mathrm{mg} / \mathrm{dL}$ (mean $\pm \mathrm{SD}$ )

LV-EF (basal), \% (mean \pm SD)

LV-EF (3rd month), \% (mean \pm SD)

\section{LV reverse remodeling}

group

$56 \pm 9.9$

$78.4 \%$

43.9

17.3

66.2

41

21.6

13.7

12.9

0.7

11.5

1.4

14.4

8.6

22.3

16.5

5.8

2.8

7.9

14.4

46.8

17.5

35

90.6

$29.5 \pm 5.4$

$131 \pm 28$

$78 \pm 18$

$76 \pm 16$

$12.2 \pm 3.1$

$13.5 \pm 1.9$

$15.4 \pm 2.4$

$264.6 \pm 66$

$0.9 \pm 0.3$

$143 \pm 69$

$179 \pm 38$

$114 \pm 34$

$39 \pm 12$

$145 \pm 105$

$46.8 \pm 7.3$

$53.9 \pm 7.8$

\section{LV non-reverse remodeling} group

$58 \pm 11$

$80.7 \%$

42.9

39.1

50.3

39.8

21.1

9.9

9.3

3.7

14.9

0.6

18.6

10.6

18.6

20.5

5

11.2

11.8

10.6

45.3

16.3

37.2

77.6

$30 \pm 6$

$128 \pm 32$

$74 \pm 19$

$76 \pm 19$

$12 \pm 4$

$13.4 \pm 2$

$17.8 \pm 3.6$

$259 \pm 58.5$

$1.0 \pm 0.5$

$161 \pm 90$

$176 \pm 40$

$112 \pm 37$

$39 \pm 12$

$138 \pm 73$

$46.6 \pm 7.6$

$45.6 \pm 9$ p

0.108

0.617

0.858

$<0.001^{\text {** }}$

$0.006^{\text {** }}$

0.825

0.922

0.315

0.316

0.085

0.388

0.478

0.322

0.574

0.431

0.381

0.762

$0.042^{*}$

$0.002^{\text {** }}$

0.254

0.956

$0.009^{\text {** }}$

0.889

0.521

0.088

0.917

0.905

0.568

$<0.001^{\text {** }}$

0.361

0.119

0.040*

0.527

0.660

0.637

0.522

0.796

$<0.001^{\text {** }}$

CABG: Coronary artery bypass grafting, ACE: Angiotensin converting enzyme WBC: White blood cell, PCI: Percutaneous coronary intervention, RDW: Red blood cell distribution width, LDL: Low-density lipoprotein, HDL: High density lipoprotein, LV-EF: Left ventricular ejection fraction, CX: Circumflex, RCA: Right coronary artery, LDA: Left anterior descending artery, SD: Standard deviation, TIMI: Thrombolysis in myocardial infarction, ASA: Acetylsalicylic acit *: $p \leq 0.05,{ }^{* *}: p \leq 0.01$ 
dysfunction. The mechanism underlying LV dysfunction, the most important factor determining the prognosis in the period after myocardial infarction, is LV remodeling ${ }^{(20)}$. The association between LV reverse remodeling and improvement in clinical outcomes was first demonstrated

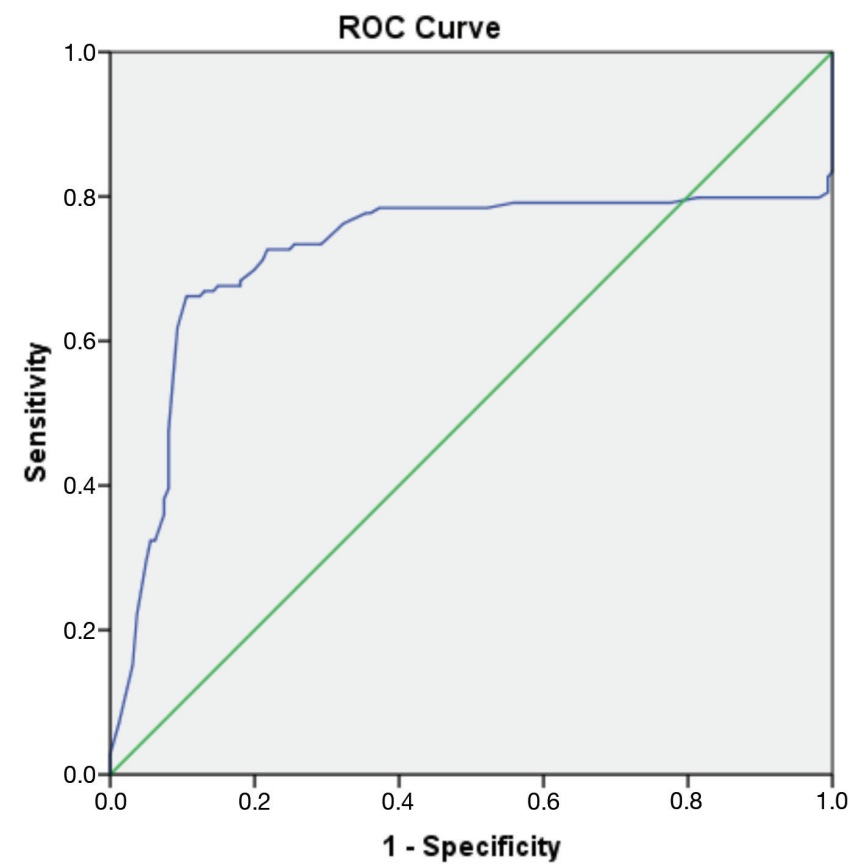

Figure 1. The ROC curve of the RDW in prediction of LV nonreverse remodeling

ROC: Receiver operating characteristic, RDW: Red blood cell distribution width, LV: Left ventricular

Table 2. Independent variables detected in the logistic regression analysis in the prediction of left ventricular nonreverse remodeling

\begin{tabular}{|l|l|l|}
\hline Diabetes Mellitus & OR $(\mathbf{9 5 \%} \mathbf{C I})$ & $\mathbf{p}$ \\
\hline Smoking & $3.4(1.7-6.7)$ & 0.001 \\
\hline History of CABG operation & $0.7(0.4-1.2)$ & 0.241 \\
\hline Killip Classification & $11.9(1.2-13.2)$ & 0.031 \\
\hline RDW & $1.4(0.6-3)$ & 0.319 \\
\hline Diastolic blood pressure & $1.30(1.20-1.43)$ & $<0.001$ \\
\hline Glucose & $0.99(0.97-1.0)$ & 0.165 \\
\hline ST Segment resolution & $0.99(0.99-1.0)$ & 0.498 \\
\hline TIMI & $0.514(0.29-0.90)$ & 0.021 \\
\hline
\end{tabular}

CABG: Coronary artery bypass grafting; RDW: Red blood cell distribution width; TIMI: Thrombolysis in Myocardial Infarction, OR: Odds ratio, Cl: Confidenceinterval by Pfeffer et al. ${ }^{(21)}$, who determined that angiotensinconverting enzyme inhibition improved survival after AMI by providing LV reverse remodeling. Additionally, a multicenter trial by Daubert et al. ${ }^{(19)}$ similarly showed the association among increase of EF, reverse LV remodeling and mortality. Furthermore, the level of LV remodeling was determined to be correlated with the risk of death and HF events in the Echo sub-study analysis of the VALIANT study $^{(22)}$. This value of EF change was determined to be correlated with reduced mortality in similar patients with reduced $\mathrm{EF}^{(23)}$. In our study, LV reverse remodeling was defined as improved systolic function with an increase of 5\% and higher in EF from the baseline to follow-up 3 months later.

It is well known that the inflammatory process plays a crucial role in LV reverse remodeling following AMI that causes the activation of an inflammatory response ${ }^{(24)}$. The association of RDW with inflammatory processes has also been demonstrated in previous studies ${ }^{(25)}$. Furthermore, there is growing evidence that RDW is associated with HF, metabolic syndrome and poor prognosis of coronary artery disease ${ }^{(26-29)}$. In this study, we found that RDW, one of the simple and inexpensive inflammatory biomarkers in clinical practice, was independently associated with

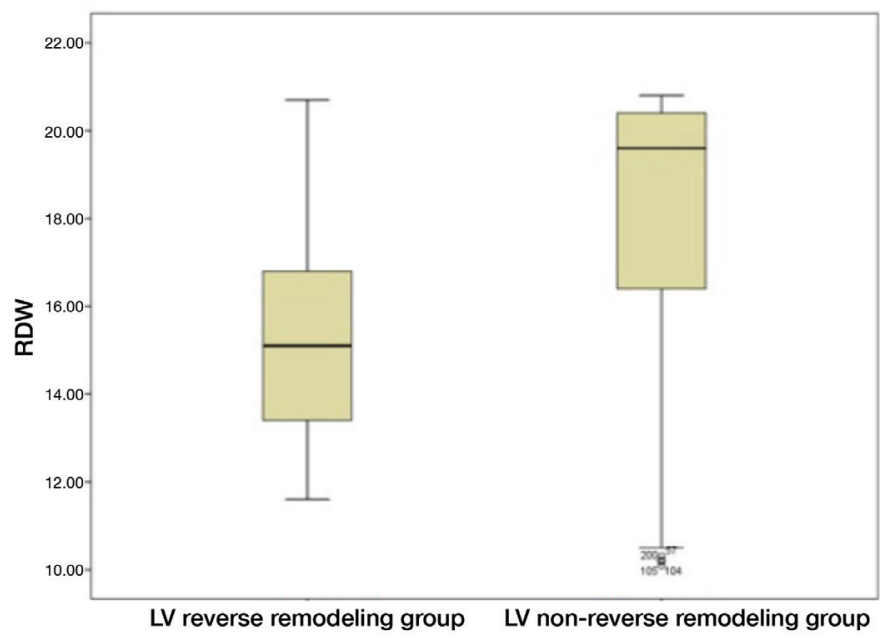

Figure 2. The mean RDW value was higher in the LV nonreverse remodeling group than in the $L V$ reverse remodeling group (17.8 \pm 3.6 vs $15.4 \pm 2.4, \mathrm{p}<0.00)$

RDW: Red blood cell distribution width, LV: Left ventricular 
LV reverse remodeling. According to the review of Fertin et al. ${ }^{(30)}$, numerous circulating biomarkers such as white blood cell, neutrophil lymphocyte ratio, C-reactive protein, brain natriuretic peptide, atrial natriuretic peptide, neopterin and aldosterone have been reported as independent predictive markers in LV reverse remodeling after AMI. It is possible to say that our results are consistent with the literature when we consider that most of the biomarkers and RDW are related to inflammatory processes. This is thought to be a possible mechanism in explaining the RDW and LV reverse remodeling relationship.

Another possible mechanism for explaining the association of RDW and LV reverse remodeling is focused on the structure of membranous cholesterol on erythrocytes. Membranous cholesterol on erythrocytes is a source of atherosclerotic nuclei, and it is being discussed that erythrocyte membrane cholesterol levels may be a new marker in the diagnosis of acute coronary syndrome ${ }^{(30)}$. Tziakas et al. ${ }^{(31)}$ demonstrated that RDW and erythrocyte membrane cholesterol levels were correlated with each other. These data show that RDW levels may be used to demonstrate plaque instability.

We also found that the presence of DM, CABG operation history, ST segment depression rate after PPCI and TIMI flow class could be used in STEMI patients, as well as baseline RDW level as a predictor of LV reverse remodeling ${ }^{(32)}$. It is possible to state that these data were in accordance with the literature. Moreover, our study found that smoking, ST segment resolution and glucose levels were associated with LV reverse remodeling development in our patient population.

\section{Study Limitations}

The most important limitation of our study was that it was retrospective, and it may be stated that providing more accurate data is an obstacle. Additionally, the number of patients included in the study was limited, and the echocardiography controls performed 3 months after the hospitalization of patients may lead to resultant variability due to the short follow-up period. The current data have shown that there may be improvement over a period of one year after AMI. Additionally, the short- and long-term morbidity and mortality rates of the patients were not evaluated, and the relationship of the results with survival was not mentioned. The use of echocardiography in evaluation of LV reverse remodeling and healing was also a major limitation. Today's gold standard imaging method for assessing LV function is cardiac MR imaging. It should be noted that echocardiographic imaging at this point may lead to incorrect results.

\section{Conclusion}

Measuring initial RDW levels may be useful for predicting LV reverse remodeling in patients with STEMI who are treated with PPCI. RDW may be helpful in making adverse cardiovascular event risk assessments of patients as an objective value that can be measured simply and quickly. Studies with a larger sample size are needed to plan to further investigate serum RDW levels in LV reverse remodeling after AMI.

\section{Ethics}

Ethics Committee Approval: Ethics committee approval was received for this study from the Clinical Trials Ethics Committee of Atatürk University (decision no and date: $4 / 17$ and 02.06.2015).

Informed Consent: Retrospective study.

Peer-review: Internally and externally peer-reviewed.

\section{Authorship Contributions}

Concept: P.D.G., Design: P.D.G., K.K., E.A., Data Collection or Processing: P.D.G., R.D., K.K., Analysis or Interpretation: E.A., A.U., Literature Search: P.D.G., R.D., Writing: E.A., R.D.

Conflict of Interest: The authors declare no potential conflicts of interest with respect to the research, authorship, and/or publication of this article. This article was adapted from the corresponding author's specialty thesis. 
Financial Disclosure: The authors received no financial support for the research, authorship, and/or publication of this article.

\section{References}

1. Mozaffarian D, Benjamin EJ, Go AS, Arnett DK, Blaha MJ, Cushman M, et al. Heart disease and stroke statistics - 2016 update: a report from the American Heart Association. Circulation 2016;133:e38-e360.

2. Cohn JN, Ferrari R and Sharpe N. Cardiac remodeling-concepts and clinical implications: a consensus paper from an international forum on cardiac remodeling. Journal of the American College of Cardiology 2000;35:569-82.

3. Parodi $G$ and Antoniucci D. Left ventricular remodeling after primary percutaneous coronary intervention. American heart journal 2010;160:S11-S5.

4. Sun Y. Intracardiac renin-angiotensin system and myocardial repair/ remodeling following infarction. Journal of molecular and cellular cardiology 2010;48:483-9.

5. Levin HR, Oz MC, Chen JM, Packer M, Rose EA, Burkhoff D. Reversal of chronic ventricular dilation in patients with end-stage cardiomyopathy by prolonged mechanical unloading. Circulation 1995;91:2717-20.

6. Solomon SD, Skali H, Anavekar NS, Bourgoun M, Barvik S, Ghali JK, et al. Changes in ventricular size and function in patients treated with valsartan, captopril, or both after myocardial infarction. Circulation 2005;111:3411-9.

7. Tarantini G, Razzolini R, Cacciavillani L, Bilato C, Sarais C, Corbetti F, et al. Influence of transmurality, infarct size, and severe microvascular obstruction on left ventricular remodeling and function after primary coronary angioplasty. The Am J Cardiol 2006;98:1033-40.

8. Evans TC, Jehle D. The red blood cell distribution width. J Emerg Med 1991;9:71-4.

9. Lappé JM, Horne BD, Shah SH, et al. Red cell distribution width, C-reactive protein, the complete blood count, and mortality in patients with coronary disease and a normal comparison population. Clinica Chimica Acta 2011;412:2094-9.

10. Shao Q, Li L, Li G, Liu T. Prognostic value of red blood cell distribution width in heart failure patients: a meta-analysis. Int J Cardiol 2015;179:4959.

11. Chang X-W, Zhang S-Y, Wang H, et al. Combined value of red blood cell distribution width and global registry of acute coronary events risk score on predicting long-term major adverse cardiac events in STEMI patients undergoing primary PCI. Oncotarget 2018;9:13971.

12. Yazııı S, Kırış T, Sadık Ceylan U, et al. Relation between dynamic change of red cell distribution width and 30-day mortality in patients with acute pulmonary embolism. Clin Respir J 2018;12:953-60.

13. Bozorgi A, Mehrabi Nasab E, Khoshnevis M, Dogmehchi E, Hamze G and Goodarzynejad H. Red cell distribution width and severe left ventricular dysfunction in ischemic heart failure. Crit Pathw Cardiol 2016;15:174-8.

14. Karakas MS, Korucuk N, Tosun V, Altekin RE, Koç F, Ozbek SC, et al. Red cell distribution width and neutrophil-to-lymphocyte ratio predict left ventricular dysfunction in acute anterior ST-segment elevation myocardial infarction. J Saudi Heart Assoc 2016;28:152-8.

15. Thygesen K, Alpert JS, Jaffe AS, Chaitman BR, Bax JJ, Morrow DJ, et al. Fourth universal definition of myocardial infarction (2018). J Am Coll Cardiol 2018:25285.

16. Antman EM. ST-elevation myocardial infarction: pathology, pathophysiology, and clinical features. Heart Disease 2008:1207-32.

17. Gibson CM, Cannon CP, Daley WL, DodgeJr JT, Alexander B, Marble SJ, et al. TIMI frame count. Circulation 1996; 93:879-88.

18. Huttin O, Coiro S, Selton-Suty C, Juillière Y, Donal E, Magne J, et al Prediction of left ventricular remodeling after a myocardial infarction: role of myocardial deformation: a systematic review and meta-analysis. PloS one 2016;11:e168349.

19. Daubert MA, Massaro J, Liao L, Pershad A, Mulukutla S, Ohman EM, et al. High-risk percutaneous coronary intervention is associated with reverse left ventricular remodeling and improved outcomes in patients with coronary artery disease and reduced ejection fraction. Am J Heart 2015; $170: 550-8$

20. Pfeffer MA, Braunwald E. Ventricular remodeling after myocardial infarction. Experimental observations and clinical implications. Circulation 1990;81:1161-72.

21. Pfeffer MA, Braunwald E, Moyé LA, Basta L, Brown EJ, Coddy TE, et al. Effect of captopril on mortality and morbidity in patients with left ventricular dysfunction after myocardial infarction: results of the Survival and Ventricular Enlargement Trial. N Eng J Med 1992;327:669-77.

22. Scott D, Solomon S, Skali H. Changes in Ventricular Size and Function in Patients Treated with Valsartan, Captopril, or Both After Myocardial Infarction. ACC Curr J Rev 2005;10:3.

23. Kramer DG, Trikalinos TA, Kent DM, Antonopoulos GV, Konstam MA, Udelson JE. Quantitative evaluation of drug or device effects on ventricular remodeling as predictors of therapeutic effects on mortality in patients with heart failure and reduced ejection fraction: a meta-analytic approach. J Am Coll Cardiol 2010;56:392-406.

24. Seropian IM, Toldo S, Van Tassell BW, Abbate A. Anti-inflammatory strategies for ventricular remodeling following ST-segment elevation acute myocardial infarction. J Am Coll Cardiol 2014;63:1593-603.

25. Lippi G, Targher G, Montagnana M, Salvagno GL, Zoppini G, Guidi GC. Relation between red blood cell distribution width and inflammatory biomarkers in a large cohort of unselected outpatients. Archives of pathology \& laboratory medicine 2009;133:628-32.

26. Osadnik T, Strzelczyk J, Hawranek M, Lekston A, Wasilewski J, Kurek A et al. Red cell distribution width is associated with long-term prognosis in patients with stable coronary artery disease. BMC cardiovascular disorders $2013 ; 13: 113$

27. Sánchez-Chaparro MA, Calvo-Bonacho E, González-Quintela A, Cabrera M, Sáinz JC, Fernández-Labandera $\mathrm{C}$, et al. Higher red blood cell distribution width is associated with the metabolic syndrome. Diabetes Care 2010;33:e40-e.

28. Allen LA, Felker GM, Mehra MR, Chiong JR, Dunlap SH, Ghali JK, et al. Validation and potential mechanisms of red cell distribution width as a prognostic marker in heart failure. J Card Fail 2010;16:230-8. 
29. Özcan F, Turak O, Avci S, Tok D, İşleyen A, Aras D, et al. Heart rate variability and red cell distribution width in patients with systolic left heart failure. Scand Cardiovasc J 2013;47:225-9.

30. Fertin M, Dubois E, Belliard A, Amouyel P, Pinet F, Bauters C. Usefulness of circulating biomarkers for the prediction of left ventricular remodeling after myocardial infarction. Am Journal Cardiol 2012;110:277-83.
31. Tziakas D, Chalikias G, Grapsa A, Gioka T, Tentes I, Konstantinides S. Red blood cell distribution width-a strong prognostic marker in cardiovascular disease-is associated with cholesterol content of erythrocyte membrane. Clin Hemorheol Micro 2012;51:243-54.

32. Akboga MK, Yayla C, Yilmaz S, Sen F, Gulcihan Balci K, Ozcan F, et al. Increased red cell distribution width predicts occlusion of the infarctrelated artery in STEMI. Scand Cardiovasc J 2016;50:114-8. 